\title{
Simon Coffey, ed. by (2021). The History of Grammar in Foreign Language Teaching...
}

\section{Henri Besse}

\section{(2) OpenEdition \\ 1 Journals}

\section{Édition électronique}

URL : https://journals.openedition.org/dhfles/8623

DOI : $10.4000 /$ dhfles.8623

ISSN : 2221-4038

Éditeur

Société Internationale pour l'Histoire du Français Langue Étrangère ou Seconde

Édition imprimée

Date de publication : 1 décembre 2021

ISSN : 0992-7654

\section{Référence électronique}

Henri Besse, «Simon Coffey, ed. by (2021). The History of Grammar in Foreign Language Teaching... », Documents pour l'histoire du français langue étrangère ou seconde [En ligne], 66-67 | 2021, mis en ligne le 02 février 2022, consulté le 25 mars 2023. URL : http://journals.openedition.org/dhfles/8623 ; DOI : https://doi.org/10.4000/dhfles.8623

Ce document a été généré automatiquement le 25 mars 2023.

Tous droits réservés 


\title{
Simon Coffey, ed. by (2021). The History of Grammar in Foreign Language Teaching...
}

\author{
Henri Besse
}

1 Simon Coffey, ed. by (2021). The History of Grammar in Foreign Language Teaching. Collection Language and Culture in History. Amsterdam : Amsterdam University Press, 254 pages.

2 L'ouvrage comprend douze chapitres (numérotés de 1 à 12), précédés d'une introduction par Simon Coffey et suivis d'une postface de Nicola McLelland. Coffey a réussi l'exploit de le concevoir et de le mettre en oeuvre en orientant quatorze contributions, rédigées par dix-huit auteur(e)s, non sur la grammaire en elle-même et pour elle-même, mais sur l'art d'enseigner / apprendre les langues qui a façonné, depuis plus de deux millénaires, ce savoir grammatical et partant la culture lettrée occidentale. Chaque contribution est suivie de sa bibliographie et d'une brève présentation de son auteur(e) ; un Index, des noms et des notions, clôt l'ouvrage.

Le grand mérite de cet ensemble est de questionner, de points de vue très différents, l'histoire de l'élaboration d'un savoir grammatical qui y apparaît comme non dissociable de l'enseignement / apprentissage des langues, qu'elles soient natives ou non. On suivra l'ordre, en gros historique, de ces douze chapitres, en veillant à ne pas trop répéter Coffey \& McLelland.

4 1. «The emergence of grammar in the Western world » par Pierre Swiggers \& Alfonso Wouters (23-42).

5 Pour Swiggers \& Wouters, ce ne sont ni les rhéteurs ni les philosophes qui ont donné de la grammaire « a proper definition » (26), celle-ci s'étant constituée, au III ${ }^{\mathrm{e}}$ siècle av. J.-C., (je souligne) " in constant interaction with rhetorical and philosophical views as well as with didactic concerns » (24). Pour étayer leur thèse, Swiggers \& Wouters débutent leur chapitre en citant, entre autres, un passage du début de la Tekhnê de Denys le Thrace qui en fait un savoir pratique lié à l'enseignement de la littérature grecque antérieure à l'époque ptolémaïque. 
Cette Tekhnê est, selon eux, " at the beginning of a 'manualistic' tradition, represented by a number of preserved papyrus texts » (29), allant du $\mathrm{I}^{\mathrm{er}}$ au $\mathrm{V}^{\mathrm{e}}$ siècle apr. J.-C., textes ayant pour fonction d'établir et de renforcer « the terminological and criterial frame for the work of the grammarian, consistant in reading, explaining, and commenting texts » (31). Soit un savoir élaboré (souligné par eux) «in a school context», sans quoi les huit parties du discours d'alors ne seraient pas ce qu'elles sont encore de nos jours.

7 La dernière partie de leur chapitre est intitulée: "Exercising one's grammatical knowledge ». Elle est consacrée, pour l'essentiel, à « an intriguant type of exercise [...] klisis chreias, which consisted in starting from a sentence, and turning the verbs and nouns into the possible numbers (from singular to dual and plural) and cases " (35). Exercice connu, dans le monde romain, "as exercitatio chriarum», constitutif des "preliminary exercises' (progynasmata) in rhetoric » mais exercices qui sont « overtly grammatical » (36), soit cette chria ou chrie qui hante l'histoire de la didactique occidentale des langues.

2. et 3. "Secondary Grammar Education in Middle Ages" par Anneli Luthala; " Grammar is the key, Aelfric's Grammar and the teaching of Latin in Tenth-Century England » par Don Chapman (63-76).

Luthala traite de l'enseigner/apprendre le latin en latin, de l'antiquité tardive à la fin du Moyen Age. Seuls les garçons apprenaient, autour de sept ans, à oraliser les lettres latines et à les écrire tout en mémorisant quelques textes latins (dont les Psaumes) ; «at the secondary level of instruction they progressed to the meticulous analysis of the elements of their own mother tongue, outlined in basic textbook on grammar » (44).

10 Les écoles romaines ayant disparu au VII ${ }^{e}$ siècle, celles des monastères et des cathédrales leur succédèrent. "Latin was the lingua franca which each generation had to acquire anew in order to be able to take part in the various functions of society, educational institutions and the Church.» (ibid.) Dans ces «grammarian's schools» (46), deux manuels pour enseignants dominaient: l'Ars minor de Donat (c. 360) et les Institutiones grammaticae de Priscien (526-527). Le Donat énonçait les huit parties du discours dans un ordre ; le Priscien, écrit à l'origine pour des élèves hellénophones et divisé plus tard en Priscianus maior (livres I-XVI) et Priscianus minor (livres XVII-XVIII), s'en tenait à sept parties ordonnées autrement que dans le Donat.

11 Le pape Grégoire le Grand (ca. 540-604) jugeait que "the sacred texts should not be subjected to Donatus'rules» (49), mais Donat avait, lui, «the advantage of being the teacher of St. Jerome, the translator of the Bible into Latin, which recommended Donatus's grammar for use in medieval classroom » (ibid.). Quant à Priscien, redécouvert à l'époque carolingienne conjointement aux Categories et au De interpretatione d'Aristote, il suscita " a new philosophical orientation of grammatical studies » (52) aux XI ${ }^{\mathrm{e}}$ et XII ${ }^{\mathrm{e}}$ siècles. D'où " the famous Battle of Arts [...] between the traditional literary orientation in grammar teaching, which had flourished in Chartres and Orléans, and the new logic-oriented teaching, pursued especially in the Parisian schools $»(53)$.

12 C'est le manuel anglo-saxon bilingue d'un moine dénommé Aelfric, intitulé Excerptiones de arte grammatica angelic (« Extraits sur l'art grammatical, en anglais », ca. 992), qui est l'objet de la contribution de Chapman. Ces « extraits » sont empruntés à Priscien plus qu'à Donat et sont expliqués en vieil anglais par Aelfric, traduits en anglais actuel par Chapman. Pour lui, « one of Aelfric's clearest statements about the purpose of grammar comes toward the end of his grammar: 'Grammar is skill with letters. This skill opens and upholds the 
Latin language, and no man has a full understanding of Latin books unless he knows that skill.' » (66) D'où le titre de la contribution de Chapman : Grammar is the key.

13 C'est un manuel « for beginners learning Latin as a foreign language » (69), le premier de ce type : " no one had apparently done that before» (70). Une innovation qui en a entrâné d'autres: le Donat ne donnait pas les paradigmes des formes des noms et des verbes, "Aelfric gave full paradigms» (ibid.); l'Aelfric s'en tient à "five declensions [" déclinaisons»] and four conjugations» (71) et privilégie les exemples familiers aux élèves. Un premier manuel bilingue qui n'a pas eu la postérité qu'il méritait.

4. « Spanish grammaticography and the teaching of Spanish in the sixteenth century " par José J. Gomez Asencio, Carmen Quijada van den Berghe \& Pierre Swiggers (77-95).

14 Cette contribution traite du manuel d'Antonio de Nebrija intitulé Grammatica sobre la lengua castillana, la "first printed grammar of Spanish, also the first printed grammar of a modern (European) vernacular» (78). Nebrija distingue, en et sur l'espagnol (comme on dira plus tard), dix parties du discours, ajoutant au huit traditionnelles, le gérondif et le «nom participial infinitif».

Son originalité didactique est, à mes yeux, son Libro $V$, non annoncé dans la préface de la Grammatica et intitulé : Delas introduciones dela lengua castellana para los que de estrana lengua querran deprender. Soit une grammaire "for non-native speakers» (80), qui a sa propre préface où est pointée "the difference between the orden natural de la grammatica and the orden de la doctrine » (82), le « naturel » étant celui des quatre Libros initiaux de la Grammatica, le second, celui du Libro $V$, étant « a didactic order, more suited for those who start learning a language (including young native speakers who already speak the language but are in want of grammatical instruction " (ibid.). Une lecture de ce livre à laquelle les trois auteurs de cette contribution semblent tenir puisqu'ils l'ont déjà formulée à la page précédente : « one can easily imagine that Book $V$ would constitute the most adequate tool for Spanish-speaking children and youngsters to learn the Spanish language » (81).

Cette lecture du Livro $V$, qui en fait une version simplifiée des quatre premiers Livres de la Grammatica, me semble assez répandue en Espagne. La raison en est peut-être le rôle joué par cette grammaire dans l'enseignement certes de l'espagnol dans «les Espagnes », mais aussi dans "the 'Spanish Netherlands' " (83-86), en Italie (86-87), en Angleterre (87-90) et en France (90-91). Une lecture qui me paraît minimiser l'innovation proprement didactique du Libro $V$ : enseigner les catégories grammaticales sans les dénommer, à partir des seuls paradigmes censés les illustrer.

17 5. et 6. "Quelle grammaire française pour les étrangers, du seizième au dixhuitième siècles ?" par Valérie Raby ${ }^{1}$ (97-112); "Grammar in verse : Latin pedagogy in seventeenth-century England » par Victoria Moul. (113-131).

Le début de la contribution de Raby, sous-titrée La grammatisation du français et son "public", défend la thèse que les grammaires en français sur le français - de John Barton [ca 1409] à Nicolas Beauzée [1767] - étaient destinées à des « locuteurs reconnus comme linguistiquement qualifiés » et non à " ceux qui ne le sont pas, qu'ils soient 'français naturels' ou étrangers » (101): il s'agissait de "fabriquer, par sélection et stabilisation des formes, une langue commune qui n'est pas davantage celle de nombreux 'Français' qu'elle ne l'est des étrangers » (ibid.). De cette "relative indistinction fonctionnelle des premières grammaires françaises ", elle conclut, dans le droit fil des onze autres 
chapitres, que « la tâche de l'historien de la didactique des langues est de rechercher la trace des pratiques effectives d'apprentissage et d'enseignement » (102).

C'est donc « entre principes et pratiques » (ibid.) qu'elle situe sa seconde partie. Elle y inscrit les grammaires de son corpus dans un dispositif plurilingue, dont «la matrice " est la «grammatica [latine], à la fois langue et grammaire pivot, pour amener l'élève à comprendre le système du vernaculaire et à produire les formes correctes attendues » (103), une matrice servant à enseigner, par « des opérations de traduction constitutives de la grammaire latine étendue " (104), le grec et l'hébreu anciens ainsi que les vernaculaires européens. Les difficultés pratiques auxquelles s'est heurté ce «cadre théorique latin» est, selon Raby, à l'origine du «programme de la grammaire générale », de Port-Royal à Beauzée (106).

Une thèse plus proche de celle de Swiggers \& Wouters que de celle de certains des tenants français des «idées linguistiques " par trop inattentifs au didactique : «il est évident que les difficultés d'enseignement ont stimulé la réflexion de grammairiens qui, dans leur grande majorité, sont aussi des pédagogues ».

Moul s'inscrit dans un projet de recherche intitulé «"Neo-latin Poetry in Early Modern English Manuscript Sources (c. 1550-1700)' ». Soit un siècle et demi durant lequel « Latin was the medium as well as the main subject of all early modern education across Europe, in both Protestant and Catholic countries" (113). Projet focalisé sur "the widespread use of Latin verse (rather than prose) in the teaching of elementary Latin, as well of more advanced subjects, such as Ancient Greek and Hebrew » (114).

Elle s'attarde sur le Carmen de moribus de William Lily, écrit au début du XVI ${ }^{\mathrm{e}}$ siècle d'abord pour ses propres élèves mais qui fut imprimé durant des siècles parce qu'il fut "prescribed by royal authority for use in schools» en 1542 (115). Un manuel en latin versifié «on proper behavior» (ibid.), qui devint un modèle en Angleterre et hors d'Angleterre: Moul rappelle que cette pratique versifiée fut aussi celle des collèges jésuites, dont la grande majorité, sauf en France, utilisait le manuel [1572] d'« Emanuel Alvarez's De institutione Grammatica Libri III » (119).

7. «Learning grammar in eighteenth-century Russia " par Ekaterina Kislova,Tatiana Kostina \& Vladislav Rjéoutski (132-154).

24 En introduction, il est rappelé que la Russie disposait au XVIII ${ }^{e}$ siècle de trois types d'institutions pour enseigner / apprendre les langues étrangères. Deux types où les élèves étaient admis pour ainsi dire par hérédité familiale : les séminaires de l'Église orthodoxe réservés aux enfants de son clergé et les écoles destinées aux enfants de la petite et moyenne noblesse. Pierre le Grand [1672-1725] avait imposé dans les séminaires le latin (y échouer valait exclusion du clergé) mais aussi l'allemand et le français ; deux langues vulgaires qui étaient aussi enseignées dans les écoles des nobles, où elles l'emportaient sur le latin. Et un troisième type, celui des « learning institutions of the Academy of Sciences" [fondée en 1725], ouvertes à un "wide range of social groups, including children of nobility, merchants, artisans, non-commissioned officers, soldiers from guard regiments and more " (134), où l'on étudiait également l'allemand, le latin et le français. Ce qui reflète « the acceptance of the roles of these languages in the cultural outlook of all privileged social groups » (ibid.).

25 La première partie donne " a short overview of how 'grammar' was understood in Russia » (ibid.) avant le XVIII siècle. De la fin du XV à la fin du XVII ${ }^{e}$ siècle, le slavon d'église mêlé de russe de l'Église orthodoxe, écrit alors en alphabet glagolitique, était 
déjà étudié « according to the Latin or Greek model » (135). Pierre le Grand ayant imposé l'alphabet cyrillique, " until the mid-eighteenth century, 'grammar' referred to a graphic and spelling reference book proper for learning to write and read correctly, but unsuitable for mastering the morphological system of the Church Slavonic language "(ibid.). Il faudra attendre le XIX siècle pour qu'un enseignement "grammar-oriented» de ce Church Slavonic et des langues vivantes finisse par s'imposer.

Leur seconde partie esquisse un genre de socio-didactique raisonnée, à partir des archives des trois types d'institutions rappelées ci-dessus, "in terms of the national makeup of their students and faculty» (136). Dans les séminaires, la majorité des élèves et des enseignants étaient russophones. Une Spiritual Régulation [1721], inspirée de la tradition de l'Académie de Kiev et de la Ratio Studiorum jésuite, devint " the main guideline for church education until the beginning of the nineteenth century: Instruction [...] was given either primarily or entirely in Latin» (137), les autres langues étrangères français, allemand, grec, hébreu - étant apprises à titre optionnel. Dans les écoles des Cadets et surtout dans celles de l'Académie des sciences, une proportion significative des étudiants venait de familles germanophones et la majeure partie des enseignants était germanophone, nombreux étant ceux qui ne parlaient pas russe. Au point qu'on finira par y apprendre la grammaire latine après avoir appris la grammaire russe, celleci servant de base à celle-là. Un enseignement qui n'était guère, au moins pour ce qui du français, celui des précepteurs privés : "many French native speaking teachers had a poor grasp of grammar » (142).

La troisième partie «deals only with the more popular grammar books» (ibid.) utilisés en Russie, dans ce même XVIII ${ }^{e}$ siècle, pour enseigner le latin, l'allemand, le français et le russe. La bibliographie atteste que la plupart de ces manuels étaient importés de l'Ouest européen.

8. "Wanostrocht's Practical Grammar and the grammar-translation model " par Simon Coffey (155-172).

Nicolas Wanostrocht (1745-1812), protestant de Bourgogne émigré en Angleterre vers 1770-1780, était « a well-educated French native-speaker » (156), précepteur d'abord dans « a noble family, celle du Lord Chancellor of Great Britain from 1771 to 1778 », avant d'ouvrir, vers 1790, sa propre école à Londres : the Alfred House Academy » (157).

29 Sa grammaire a pour titre, dans ces deux premières éditions $(1780,1782)$, A Practical Grammar of the French Language; dans les trois suivantes $(1789,1792,1795)$, A Grammar of the French Language with Practical Exercises. L'analyse qu'en fait Coffey porte sur l'édition de 1795, dont les amendements par rapport aux précédentes ne sont pas, selon lui, «substantial» (note 19).

30 Cette édition comprend neuf Sections sur les parties du discours, cinq déclinables et quatre indéclinables, suivies de 'Remarks and exercises on the words de, à, and pour', ainsi que d'extraits de textes à traduire. Coffey note que les « rules for pronunciation ${ }^{2}$ are totally omitted " (161), Wanostrocht jugeant que " the ear cannot be properly formed without the assistance of a good speaker' » (162). Tout comme y sont absentes les règles de la syntaxe, l'auteur estimant que ses neuf parties du discours, bien étudiées, rendent inutiles son enseignement, à l'exception du « 'proper usage' » de à, de et pour (ibid.).

31 Coffey en commente (160-162) un passage de sa Section I ('Of the article'), où Wanostrocht affirme d'abord que " 'Grammar is of two kinds, general and particular' ", la première étant une "'universal grammar'", la seconde appliquant ces "'common principles'» à une 
langue particulière, avant d'ajouter qu'une "'grammar of the French tongue must be formed agreeably to the established usage, and those particular modes of expression to which custom has given its sanction'». Coffey s'appuie sur quelques auteurs pour qui «that universalism (at least in its hard-line form) was in abeyance [abandonné] » à la fin du XVIII ${ }^{\mathrm{e}}$ siècle, cette option ayant, selon ces auteurs, "'inhibited attempt to develop a grammar and mode of teaching's " propre à chaque langue. Coffey en conclut que Wanostrocht tempère (mitigates) son universalisme en prêtant attention aux particularités propres à l'usage et à la coutume du français. Pour lui, Wanostrocht a conçu sa grammaire française « as a pedagogical primer rather than a complete grammar", ses Practical exercises étant complétés "by both speaking practice and extended literary translations", soit une grammaire « to be used with a native-speaking 'master' » (168).

9. "Language turned back on itself" ${ }^{4}$. Growth and structure of the English metalanguage » par John Walmsley (173-190).

Pour Walmsley, un terme métalinguistique est (souligné par lui) «a concept uniquely associated with a label » (174), définition qu'il applique aux « English terms used to describe English structures» (ibid.). Il en distingue cinq types (175-176): les "lexical categories» (celles, en gros, des parties du discours); les " attributes» présents à la fin des noms et des verbes (number, person, tense, aspect and mood) ; la "phrase structure»; les « functional categories", c'est-à-dire « the traditional functional parts of sentence, such as 'subject', 'predicate', 'adjunct', etc. »; et les "participant roles» qui dénotent "the typical parts with entities play in situations, such as the initiator of an action, the victim, etc. ». À quoi, il ajoute des termes relevant de la linguistique moderne, tels nominal aspect, deixis, inalienable possession...

Walmsley applique d'abord ce métalangage aux grammairiens anglais médiévaux ainsi qu'à ceux du Middle English (177-178); à la "William Lily's grammar of Latin in English » [1548-1549], vue comme " a major turning point between the Middle Ages and present-day grammatical terminology" (177-178); au Pamphlet for Grammar [1586] de W. Bullokar, première grammaire de l'anglais en anglais (180-181); à l'« English grammar, adapted to the different classes of learners " de L. Murray [1795] et à A Grammar of the German Language [1830] de C. F. Becker (181-182). Pour ce qui est du XX siècle (182-183), à celles d'E. A. Sonnenschein, de Ch. C. Fries [1952] et à celle d'O. Jespersen [1961]. Quant à « the 'Chomskyan Revolution'", elle a conduit à une "proliferation of terminological innovations unparalleled in the history of linguistics» (183).

Selon Walmsley, "the salient feature of English grammatical terminology is its robustness» (184): ce sont plus ou moins les mêmes catégories lexicales que celles adoptées par Lily dans sa grammaire en anglais du latin, certaines ayant été délaissées, d'autres ajoutées et quelques unes importées de Becker ou de Tesnière, mais il admet que sur bien des points, on est encore loin d'un consensus (185).

10. «La grammaire dans le mouvement de la réforme en France et en GrandeBretagne » par Javier Suso Lopez \& Irene Valdés Melguizo (191-210).

Ce chapitre a le grand mérite de questionner la thématique centrale de l'ouvrage, dont le risque est de faire oublier que, au moins jusqu'à la Renaissance, "le culte de l'usus " (l'expression est de Jean-Claude Chevalier) l'a sans doute emporté sur l'enseignement de la grammaire dans l'apprentissage effectif du latin.

Suso \& Valdés estiment que le mouvement de la réforme lancé par Wilhelm Viëtor en 1882 remettait fondamentalement en cause «la façon traditionnelle d'enseigner la 
grammaire ", et ils rappellent que nombre des tenants de cette réforme étaient d'illustres linguistes (Michel Bréal, Otto Jespersen...) ou des auteurs de manuels qui étaient aussi «fréquemment des méthodologues (Henry Sweet, Charles Schweitzer, Paul Passy, Maximilan D. Berlitz, Karl Krause...) » (191-192 et note 7).

Ils citent un tenant français de la méthode directe bien moins connu, Ernest Rochelle, pour qui la grammaire reste " 'notre régisseur général' ", lequel " 'fait appliquer le règlement en se montrant le moins possible' " (192). Se retrouve là, à mon sens, quelque chose du Libro $V$ de Nebrija, une préfiguration de la "grammaire inductive » à laquelle Suso \& Valdés consacrent la dernière partie de leur contribution : La démarche inductive en grammaire (201-206).

11. « Grammar in English schools: a century of decline and rebirth » par Richard Hudson (211-228).

Hudson estime qu'en 1920 l'enseignement de la grammaire " was already established in the teaching of both English and FL [Foreign Languages], albeit only in grammar schools» (211), l'un des rôles des enseignants d'anglais, langue maternelle, étant alors de préparer les élèves à apprendre les $F L$ dans le secondaire. En 2020, la situation lui paraît très différente, ces mêmes enseignants étant désormais peu enclins à enseigner la grammaire et ceux du secondaire (tant d'anglais que des $F L$ ) guère plus. Certes, récemment, un peu de grammaire est enseigné à tous les enfants du primaire mais " justified solely by its benefits for English writing, so the link between English grammar and FL grammar has gone» (212). Et ce, alors qu'il en va autrement dans les universités : en 1920, "there was virtually no study of grammar in our universities"; en 2020, "we have linguistics, where grammar is a lively area of research and ideas" (ibid.), mais celles-ci n'irriguent guère le primaire et le secondaire. En s'appuyant sur des rapports officiels, Hudson privilégie quatre décennies pour expliquer cette évolution.

Dans les années 1920 (212-216), le gouvernement disposait de trois rapports : un sur les $F L$, un sur les «classics [langues anciennes]» et un sur l'anglais. Trois rapports complètement séparés. D'où " a rather muddled world»: alors que "both classics and the $F L$ teachers were convinced that grammatical analysis should start in English, the English teachers were much less sure ». Et quant à la méthode directe, Hudson a une conviction opposée à celle de Suso \& Valdés : «In short, a really pure application of the Direct Method would leave no place at all for grammar teaching. »

43 Dans les années 1950 (216-223), "the School Certificate», jugé trop généraliste, fut remplacé par le "General Certificate of Education (GCE) » qui offrait " a wide range of subjects ", une réforme "criticized by many and justified by few ». Hudson, qui a vécu ces années-là comme élève, estime que « the picture was much the same as for 1920 ». Ce qui, néanmoins, lui a donné " a solid foundation in the Grammar of French, German and Latin ", même si "the striking feature of this education was the lack of comparison between languages ».

Dans les années 1980 (223-226), « the previous distinction between grammar schools (for the academic) and 'Secondary modern' schools (for the rest)» disparait avec la création des "comprehensive secondary schools", où est plus ou moins adopté le curriculum et la pédagogie des "grammars schools». Seul trait positif, selon Hudson, le développement de la linguistique dans les universités, «including (of course) applied linguistics - the subject most directly relevant to FL teaching ", lequel donna naissance à deux approches 
largement incompatibles, la première l'emportant sur la seconde : "the Communicative Approach and Language Awareness ».

Dans les années 2010 (223-226), « the single most important fact about FL teaching is that it is in crisis, and failing». Une crise qu'un rapport de 2016 cherche à résoudre mais le «National Curriculum for English» est quasi muet sur l'enseignement de la grammaire dans les écoles secondaires.

12. «Réflexion épistémologique en didactique du français langue étrangère sur la place de la grammaire de l'oralité ? " par Corinne Weber (229-244).

Weber cite d'emblée une de ses étudiantes : « 'Arrivée en France, j’étais confrontée à un français parlé différent de celui que j'avais appris dans mon pays. C'est grâce au roman 'Un homme, ça ne pleure pas' [...] que j'ai approché le français parlé avec des natifs'.» (232) Son objectif est partant de « déjouer le poids de l'idéologie du bon français » (ibid.) : «tout sujet doit pouvoir opérer des choix en toute conscience, rapidement et de manière autonome, c'est-à-dire à prendre le contrôle lorsqu'il parle et à s'autoréguler (et s'auto-corriger) » (233).

Le cœur de son article est consacré à un « regard théorique sur la notion de grammaire de l'oral » (233) où elle synthétise un de ses ouvrage ${ }^{5}$. Une grammaire qui, ne dissociant pas le linguistique du communicatif, est une « somme de micro-significations à tous les niveaux de la langue qui s'emboîtent et se juxtaposent [...] dans la mise en discours » (ibid.).

Elle termine sa contribution (241-242) par des « perspectives didactiques vivantes pour comprendre les tendances orales" qui s'appuient sur des "ressources numériques authentiques au service de la réflexivité métagrammaticale", dont ce qui suit (je souligne) : «Et si l'enseignant emploie dans la classe un langage proche du standard (pédagogiquement indispensable) rien ne doit l'empêcher de faire entrer la variation sociolinguistique, admettre que tout locuteur fait des choix lorsqu'il parle et que tout glissement qui s'en écarte n'est pas forcément fautif. Ce sont toujours des pratiques situées. »

Pour finir, il s'agit d'un ouvrage qui, par l'empan de ses questionnements et de ses apports, n'a pas à ma connaissance d'équivalent en français : il montre que l'histoire du savoir grammatical occidental est indissociable de l'histoire de la didactique des langues, tant étrangères que maternelles. Que Simon Coffey en soit remercié.

\section{NOTES}

1. Valérie Raby est décédée en 2019 et c'est sans doute un de ses ultimes écrits. Lire sa contribution au présent ouvrage ravive le regret qu'elle ne soit plus là. La bibliothèque du laboratoire Histoire, Épistémologie, Langage (HTL ) du CNRS possède, en cette fin d'année 2021, un fonds Valérie Raby avec sa bibliographie.

2. Coffey précise qu'il s'agit de the sounding of written letters.

3. Citation de Ian Michael dans The Teaching of English from the Sixteenth to 1870 (Cambridge : C. U. P., 1987). 
4. Citation non référencée qui semble être une reprise, à un mot près, du sous-titre, 'Language turned back on himself ' [sic], d'un recueil de quatorze interviews de M. A. K. Halliday paru en 2013. 5. Pour une didactique de l'oralité. Enseigner le français tel qu'il est parlé. Paris : Didier 2013.

\section{AUTEUR}

HENRI BESSE

ENS de Lyon 